\title{
Feasibility of short double-balloon enteroscopy-assisted endoscopic retrograde cholangiopancreatography in patients with surgically altered gastrointestinal anatomy: experience in a regional centre
}

\author{
SW Cheung ${ }^{1}$ *, MRCP, FHKCP, KS Cheng ${ }^{1}$, MRCP, FHKCP, WM Yip ${ }^{2}$, MRCP, FHKCP, KK Li', MBBS, FRCP
}

${ }^{1}$ Department of Medicine and Geriatrics, Tuen Mun Hospital, Tuen Mun, Hong Kong

${ }^{2}$ Department of Medicine and Geriatrics, Pok Oi Hospital, Yuen Long, Hong Kong

- A video clip showing double-balloon enteroscopyassisted endoscopic retrograde cholangiopancreatography is available at <www.hkmj.org >

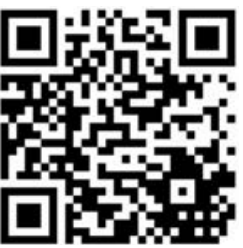

\section{Hong Kong Med J 2017;23:648-50}

DOI: 10.12809/hkmj164987

\section{Case reports}

Endoscopic retrograde cholangiopancreatography (ERCP) is a standard endoscopic technique for treating biliary obstruction and cholangitis. The presence of surgically altered gastrointestinal anatomy, however, poses a major technical difficulty to the procedure due to the long and tortuous access to the small bowel. We report a three-case series with successful attempts at short double-balloon enteroscopy (DBE)-assisted ERCP in patients with postoperative gastrointestinal anatomy. The enteroscope employed was EC-450BI5, Fujifilm endoscopy (Fig 1) and the sedation agents used in all procedures were dexmedetomidine and fentanyl continuous infusion combined with bolus midazolam.

\section{Case 1}

A 76-year-old man was admitted to our hospital in December 2015 with acute cholangitis that presented as fever and deranged liver function tests (LFT) with predominant elevation of alkaline phosphatase

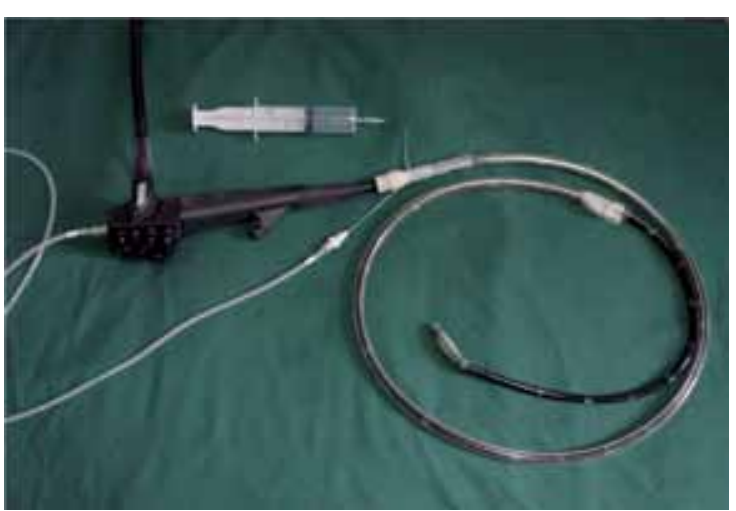

FIG I. Short double-balloon enteroscope of 152-cm working length (EC-450BI5, Fujifilm endoscopy)
(ALP). He had a history of stroke, hyperlipidaemia, and a Billroth II gastrectomy performed 30 years ago. Blood cultures grew Escherichia coli. Ultrasonography of the hepatobiliary system revealed a $5-\mathrm{mm}$ stone in the common bile duct (CBD)

The first ERCP using a standard side-view duodenoscope and end-view gastroscope failed to identify the papilla. He continued to receive antibiotic treatment, with the fever reduced but liver biochemistry remained elevated. The DBEassisted ERCP was performed 2 weeks later with an enteroscope of $152-\mathrm{cm}$ working length. The papilla was reached over the afferent limb of the small bowel by manipulation of the overtube only (no balloon inflation), followed by successful cannulation of the CBD. Cholangiogram revealed a $1.2-\mathrm{cm}$ dilated CBD with two intraductal filling defects (Fig 2a). Pre-cut papillotomy on-stent was performed with pre-insertion of a 7-French 7-cm plastic biliary stent. The plastic stent was removed after papillotomy and the papilla was dilated using a controlled radial expansion (CRE) balloon dilator of 12-mm size and subsequently stones were removed by a basket. The ERCP procedure was completed in 115 minutes and there were no complications. The patient's LFT had normalised at a follow-up 3 weeks later.

\section{Case 2}

An 80-year-old man presented in January 2016 with a history of hypertension, pulmonary fibrosis, and cerebral infarct. A Billroth II gastrectomy had been performed 10 years previously for gastrointestinal bleeding. He had an episode of acute cholangitis 4 months ago. Endoscopic retrograde cholangiopancreatography using a standard endview gastroscope failed to reach the papilla and the infection resolved after a course of antibiotic. Followup magnetic resonance cholangiopancreatography 3 weeks earlier had revealed a $1.2-\mathrm{cm}$ distal CBD stone with the $C B D$ dilated to $1.7 \mathrm{~cm}$. He then attended the 
hospital again for fever, E coli septicaemia, and an obstructive pattern of liver derangement; bilirubin level was $93 \mu \mathrm{mol} / \mathrm{L}$ and ALP level was $1224 \mathrm{U} / \mathrm{L}$. Percutaneous transhepatic biliary drainage was established and antibiotics were continued until DBE-assisted ERCP was performed 2 weeks later. With the push-pull manoeuvre, the blind end of the afferent loop was reached. Initially, a nearby minor papilla was mistaken as the major papilla and repeated attempts failed to cannulate it.

The true major papilla was then identified $5 \mathrm{~cm}$ proximal to the minor papilla and pre-cut papillotomy was performed due to difficult cannulation. The impacted CBD stone was removed following papillotomy and balloon sphincteroplasty was performed with a 12-mm CRE balloon. Subsequent cholangiogram was clear with a $1.5-\mathrm{cm}$ dilated CBD. The operating time was 163 minutes. The patient remained asymptomatic and LFT had normalised at a follow-up 1 month later.

\section{Case 3}

A female patient aged 81 years with a history of mild Parkinson's disease had undergone gastrectomy 30 years ago for peptic ulcer disease. She presented to our hospital in February 2016 for biliary pancreatitis with sudden onset of jaundice associated with epigastric pain. Amylase level was elevated to $698 \mathrm{U} / \mathrm{L}$, bilirubin level to $35 \mu \mathrm{mol} / \mathrm{L}$, and an ALP level of $1001 \mathrm{U} / \mathrm{L}$. Ultrasonography of the hepatobiliary system detected grossly dilated intrahepatic ducts (IHD) and the CBD measured up to $2.4 \mathrm{~cm}$ in diameter with one obstructing CBD stone measuring $1.5 \mathrm{~cm}$. The first gastroscopy confirmed a previous total gastrectomy with Rouxen-Y reconstruction. Percutaneous transhepatic biliary drainage was attempted but failed due to the resolution of the IHD dilatation. The patient was then managed conservatively with antibiotics and a DBE-assisted enteroscopy was scheduled 3 weeks later. The 152-cm DBE identified the blind end of the afferent limb while the endoscope advanced to $140 \mathrm{~cm}$ from the incisors after push-pull manoeuvre (Fig $2 b$ to d). Successful guidewire cannulation was followed with a cholangiogram that showed a dilated CBD with no filling defect. In view of the recent history of biliary obstruction and the possibility of a passed stone, the papilla was dilated to $13.5 \mathrm{~mm}$ with the CRE balloon and good bile drainage was observed. The operating time was 130 minutes. The major difficulty encountered was that the tip of the enteroscope was very unstable making it difficult to maintain the distal blind end and it was easily slipped out proximally. As such, repeated endoscope manipulation was required to achieve optimal positioning. Postoperatively, the patient was well, bilirubin normalised, and ALP level had reduced to $266 \mathrm{U} / \mathrm{L}$ at 1-month follow-up.

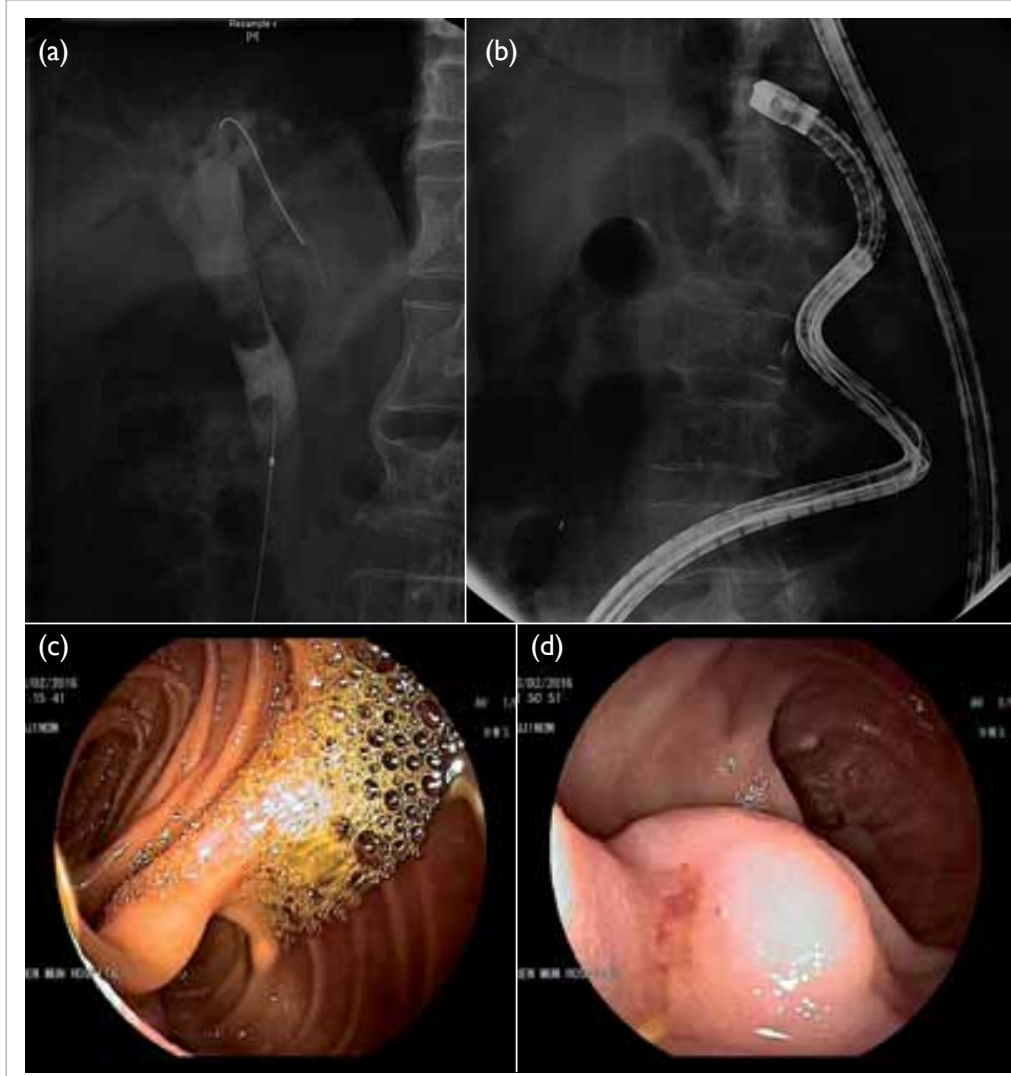

FIG 2. (a) Cholangiogram of the patient in case I showing a 1.2 -cm dilated common bile duct (CBD) with two intraductal filling defects suspicious of $C B D$ stones. (b) The ideal short position of the enteroscopy resembling a figure of ' 8 ' in deep intubation of the afferent limb of the patient in case 3 with anatomy of Roux-en-Y gastrectomy. Achieving this short position by appropriate endoscope shortening is particularly crucial to approach the papilla in enteroscopyassisted endoscopic retrograde cholangiopancreatography (ERCP) using short double-balloon enteroscopy (DBE) due to the limited length of the scope. (c) The Roux-en-Y anastomosis examined by enteroscopy. Identification of the correct pancreatobiliary limb is a major technical difficulty during the procedure. Endoscopic tattooing or clipping of the 'examined limb' may help to avoid repeated attempts to enter the same limb. (d) Locating the papilla at around 6 o'clock of the endoscopic view helps to facilitate biliary cannulation in DBE-assisted ERCP

\section{Discussion}

Balloon enteroscopy-assisted ERCP using either single-balloon enteroscopy or DBE has been reported to be an effective modality for ERCP in these patients. ${ }^{1-3}$ Traditional DBE with 200-cm length and narrow (2.2- $\mathrm{mm}$ ) accessory working channel limits the utilisation of conventional ERCP accessories. Recent attempts with a short 152-cm DBE have been highly successful. ${ }^{1,2,4}$ To date, there have been approximately 200 reports of patients who have undergone ERCP using this short DBE procedure. The success rate for reaching the blind end of the afferent limb was $86 \%$ to $100 \%$; it is not inferior to that achieved using a long-type balloon enteroscope. ${ }^{4}$ The short-type enteroscope accommodates the use 
of most available accessories to perform ERCPrelated procedures such as sphincterotomy, balloon dilatation, stone extraction, and deployment of plastic or metallic stents.

In our small series, all patients ran an uneventful course during the perioperative and postoperative period and none had any apparent complications. The overall procedural complication rate has been reported to be $8 \%$ to $10 \%$, with the rate of major complications of perforation or emphysema being around 3.5\%. ${ }^{4}$ The risk of complications, however, may vary according to different surgical approaches with consequent significant differences in the endoscopic techniques anticipated. ${ }^{3-5}$ Since it is a relatively new and evolving endoscopic technique, these rates are still experience-dependent and patients should be closely observed after the procedure.

The ERCP in the patient with Rouxen-Y reconstruction posed particular technical difficulties. The endoscopist should review previous surgical reports in detail to map and anticipate the endoscopic view at the anastomotic site. On reaching the Roux-en-Y anastomosis, identification of the pancreatobiliary limb is often difficult. After choosing either limb, the endoscopist could perform endoscopic marking by a clip or Indian ink tattoo at the entrance of the chosen limb (Fig 2c). If the chosen limb is confirmed wrong under fluoroscopy, the endoscopist can return the endoscope to the Rouxen-Y anastomosis and then insert the endoscope into the other limb. A marking at the entrance of a limb has been shown to be useful as it avoids repeated misidentification of the limb to be entered. ${ }^{6}$

Barotrauma is the major cause of intestinal perforations and may be a result of excessive air insufflation forming a closed loop between the blind end and the inflated overtube or enteroscope balloon. ${ }^{7}$ Use of carbon dioxide insufflation instead of air insufflation may reduce the chance of this closed-loop phenomenon. Additionally, use of a transparent hood at the tip of the enteroscope with instillation of water into the intestinal lumen has also been suggested to maintain the endoscopic view without gas insufflation and avoid consequent barotrauma. $^{8}$

With regard to the cannulation techniques, the catheter exits from a 7 o'clock direction of the enteroscope during DBE-assisted ERCP.
Endoscopists should attempt to locate the papilla at 6 o'clock of the endoscopic view to facilitate biliary cannulation (Fig 2b). In case of an unstable endoscopic manoeuvre, an inflated enteroscope balloon may help to grip the intestine and stabilise the manipulation. In cases where the papilla is located at 11 to 12 o'clock in the endoscopic view, the overtube balloon should remain inflated and the enteroscope rotated to solve difficult cannulation. ${ }^{6}$

In conclusion, short DBE-assisted ERCP is a safe and effective endoscopic method to treat patients with surgically altered anatomy and biliary conditions. Nonetheless local experience in enteroscopy-assisted ERCP, particularly using the short DBE, is scant because of the unavailability of the endoscopic devices. Further discussion and training opportunities are encouraged to consolidate experience and minimise procedural complications in future practice.

\section{References}

1. Moreels TG. Altered anatomy: enteroscopy and ERCP procedure. Best Pract Res Clin Gastroenterol 2012;26:34757.

2. Cheng CL, Liu NJ, Tang JH, et al. Double-balloon enteroscopy for ERCP in patients with Billroth II anatomy: results of a large series of papillary large-balloon dilation for biliary stone removal. Endosc Int Open 2015;3:E21622.

3. Mönkemüller K, Bellutti M, Neumann H, Malfertheiner P. Therapeutic ERCP with the double-balloon enteroscope in patients with Roux-en-Y anastomosis. Gastrointest Endosc 2008;67:992-6.

4. Kato H, Tsutsumi K, Harada R, Okada H, Yamamoto K. Short double-balloon enteroscopy is feasible and effective for endoscopic retrograde cholangiopancreatography in patients with surgically altered gastrointestinal anatomy. Dig Endosc 2014;26 Suppl 2:130-5.

5. Katanuma A, Yane K, Osanai M, Maguchi H. Endoscopic retrograde cholangiopancreatography in patients with surgically altered anatomy using balloon-assisted enteroscope. Clin J Gastroenterol 2014;7:283-9.

6. Hatanaka H, Yano T, Tamada K. Tips and tricks of doubleballoon endoscopic retrograde cholangiopancreatography (with video). J Hepatobiliary Pancreat Sci 2015;22:E28-34.

7. De Koning M, Moreels TG. Comparison of double-balloon and single-balloon enteroscope for therapeutic endoscopic retrograde cholangiography after Roux-en-Y small bowel surgery. BMC Gastroenterol 2016;16:98.

8. Yamamoto H. Be aware of the fatal risk of air embolism. Dig Endosc 2014;26:23. 